\title{
Prevalence of age related maculopathy in a representative Japanese population: the Hisayama study
}

\author{
Yuji Oshima, Tatsuro Ishibashi, Toshinori Murata, Yoshihisa Tahara, Yutaka Kiyohara, \\ Toshiaki Kubota
}

\begin{abstract}
Aim-To determine the prevalence of age related maculopathy (ARM) in a representative older Japanese population. Methods-1486 residents of Hisayama town, Fukuoka, Japan, aged 50 years or older were examined and the presence of ARM was determined by grading from fundus examination by indirect ophthalmoscope, slit lamp, and colour fundus photographs.

Results-The prevalence rate of drusen, which occurred with comparable frequency in men and women, was $9.6 \%$. The frequency of drusen increased with age ( $p$ $<0.01)$. Hyperpigmentation and/or hypopigmentation of the retina was present in $3.2 \%$, geographic atrophy in $0.2 \%$, and neovascular age related macular degeneration in $0.67 \%$. The frequency of neovascular age related macular degeneration was significantly higher in the men $(1.2 \%$ $v 0.34 \%, \mathrm{p}<0.01)$.

Conclusions-Early and late stage ARM is less common among Japanese people than among white people in Western countries, while late stage ARM is more common among Japanese than among black people. (Br F Ophthalmol 2001;85:1153-1157)
\end{abstract}

Department of

Ophthalmology,

Faculty of Medicine,

Kyushu University,

Fukuoka, Japan

Y Oshima

T Ishibashi

T Murata

Y Tahara

T Kubota

Second Department of Internal Medicine

Y Kiyohara

Correspondence to:

Tatsuro Ishibashi, MD,

Department of

Ophthalmology, Faculty of

Medicine, Kyushu

University, 3-1-1 Maidashi,

Higashi-ku, Fukuoka

812-8582, Japan

ishi@eye.med.kyushu-u.ac.jp

Accepted for publication 30 April 2001

Late stage age related maculopathy (ARM), especially neovascular age related macular degeneration (AMD), is the major cause of blindness and severe vision loss in older people in Western countries. ${ }^{1}$ Though not entirely clear, the pathogenesis of AMD is attributed to ingrowth of choroidal neovascularisation in the fovea, with resultant exudation, haemorrhage, and scarring. ${ }^{2}$ A number of population based epidemiological studies have been performed in Western countries to determine the prevalence, pathogenesis, and characteristics of ARM..$^{3-6}$ Although a number of studies based on population based data report the prevalence of ARM in several races and ethnic groups, including white, black, and Hispanic people, ${ }^{7-10}$ there are few data on the prevalence of ARM in Asians and none on its prevalence among Japanese people. ${ }^{11} 12$
The Hisayama study is a prospective cohort study carried out since 1961 in Hisayama, a town adjacent to Fukuoka City, a metropolitan area of Kyushu Island in southern Japan. The population of the town is approximately 7000 and has been stable for 30 years. According to the 1985 census, the age and occupational distributions of the Hisayama population were almost identical to those of Japan as a whole. The dietary pattern of the residents was also similar to that of the national nutrition survey, which selected its subjects from 300 areas throughout Japan. ${ }^{13}$ The focus of the study is the epidemiology of cerebrovascular disease and diabetes mellitus. ${ }^{14}$ We gathered ophthalmic data for this cohort in 1998, and report here, to our knowledge for the first time, the prevalence in a Japanese population of the typical features of ARM.

\section{Patients and methods}

STUDY POPULATION

From July to September 1998 we conducted a cross sectional eye examination among Hisayama residents who were at least 50 years old and who had not been under long term medical care for various chronic diseases. Among the total of 3035 residents in this age group, a baseline group of 1844 individuals (729 men and 1115 women) consented to participate in the study. Of these, 1486 (596 men and 890 women) underwent the following
OPHTHALMIC EXAMINATION

Each participant underwent a comprehensive ophthalmic examination that included automated refraction (ARK-900, Nidek, Aichi, Japan), non-contact tonometry (NT-2000, Nidek), lens grading at the slit lamp, stereoscopic fundus examination using an indirect ophthalmoscope, and a slit lamp biomicroscope with a "superfield lens" (Volk, Mentor, $\mathrm{OH}$, USA) after pupil dilatation with $1.0 \%$ tropicamide and 10\% phenylephrine. Forty five degree fundus photographs were taken using the Topcon "non-mydriatic" TRC NW-5 fundus camera (Topcon, Tokyo) and Fujichrome slide film (Sinsia II Fujifilm Co, Tokyo). The photographs were taken according to a previously described method with a 
Table 1 Characteristics of participants in the Hisayama study

\begin{tabular}{lll}
\hline Characteristic & Mean & SD \\
\hline Age (years) & 65.2 & 8.9 \\
Systolic blood pressure (mm Hg) & 137.2 & 21.8 \\
Diastolic blood presure (mm Hg) & 78.1 & 10.8 \\
$\begin{array}{l}\text { Percentage body fat } \\
\left.\text { Body mass index (kg/m }{ }^{2}\right)\end{array}$ & 24.8 & 7.1 \\
& 23.2 & 3.2 \\
\hline & No & $\%$ \\
\hline Sex & & \\
$\quad$ Female & & \\
$\quad$ Male & 890 & 59.9 \\
Cigarette smoking & 596 & 40.1 \\
$\quad$ Absent & & \\
$\quad$ Present & 1217 & 81.9 \\
Diabetic status & 269 & 18.1 \\
$\quad$ Absent & & \\
$\quad$ Present & 1215 & 81.8 \\
\hline
\end{tabular}

Table 2 Presence of any retinal drusen by age, sex, and eye in the Hisayama study, after excluding patients with worse stage of age related maculopathy in either eye

\begin{tabular}{|c|c|c|c|c|}
\hline & & & Either eye & Both eyes \\
\hline & Age (years) & No & $\%$ Freq & $\%$ Freq \\
\hline Female & $\begin{array}{l}50-59 \\
60-69 \\
70-79 \\
80+ \\
\text { Total }\end{array}$ & $\begin{array}{l}285 \\
334 \\
211 \\
58 \\
889\end{array}$ & $\begin{array}{l}6.3 \\
6.9 \\
15.1 \\
25.9 \\
9.9\end{array}$ & $\begin{array}{l}2.5 \\
4.8 \\
11.8 \\
20.7 \\
6.7\end{array}$ \\
\hline Male & $\begin{array}{l}50-59 \\
60-69 \\
70-79 \\
80+ \\
\text { Total }\end{array}$ & $\begin{array}{l}155 \\
231 \\
180 \\
32 \\
597\end{array}$ & $\begin{array}{l}5.8 \\
6.9 \\
12.2 \\
21.9 \\
9.0\end{array}$ & $\begin{array}{l}3.9 \\
3.0 \\
6.1 \\
18.8 \\
5.0\end{array}$ \\
\hline All & $\begin{array}{l}50-59 \\
60-69 \\
70-79 \\
80+ \\
\text { Total }\end{array}$ & $\begin{array}{l}440 \\
565 \\
391 \\
90 \\
1486\end{array}$ & $\begin{array}{l}6.1 \\
6.9 \\
13.8 \\
24.4 \\
9.6\end{array}$ & $\begin{array}{l}3.0 \\
4.1 \\
9.2 \\
20.0 \\
6.1\end{array}$ \\
\hline
\end{tabular}

minor modification. ${ }^{4915}$ Briefly, photographs were taken of a field centred horizontally and vertically on a point mid way between the temporal edge of the optic disc and the fovea. The photographic image included an area above and below the temporal arcades and areas just nasal to the disc and temporal to the macula. The $35 \mathrm{~mm}$ slide transparencies were mounted in clear plastic sheets and graded at Kyushu University by two experienced graders.

Table 3 The presence of each type of drusen by age and sex in the Hisayama study, after excluding patients with worse stage of age related maculopathy in either eye

\begin{tabular}{|c|c|c|c|c|c|c|c|}
\hline \multirow[b]{2}{*}{ Type of drusen } & \multirow{2}{*}{$\begin{array}{l}\text { Age } \\
\text { (years) }\end{array}$} & \multicolumn{2}{|c|}{ Female } & \multicolumn{2}{|l|}{ Male } & \multicolumn{2}{|l|}{ Total } \\
\hline & & No & $\%$ Freq & No & $\%$ Freq & No & $\%$ Freq \\
\hline Hard & $\begin{array}{l}50-59 \\
60-69 \\
70-79 \\
80+ \\
\text { Total }\end{array}$ & $\begin{array}{l}285 \\
334 \\
211 \\
58 \\
889\end{array}$ & $\begin{array}{l}0 \\
0.30 \\
0.94 \\
0 \\
0.34\end{array}$ & $\begin{array}{l}155 \\
231 \\
180 \\
32 \\
597\end{array}$ & $\begin{array}{l}0.65 \\
0.43 \\
1.1 \\
0 \\
0.67\end{array}$ & $\begin{array}{l}440 \\
565 \\
391 \\
90 \\
1486\end{array}$ & $\begin{array}{l}0.23 \\
0.35 \\
1.0 \\
0 \\
0.47\end{array}$ \\
\hline Soft distinct & $\begin{array}{l}50-59 \\
60-69 \\
70-79 \\
80+ \\
\text { Total }\end{array}$ & $\begin{array}{l}285 \\
334 \\
211 \\
58 \\
889\end{array}$ & $\begin{array}{l}6.3 \\
5.7 \\
13.7 \\
25.9 \\
9.1\end{array}$ & $\begin{array}{l}155 \\
231 \\
180 \\
32 \\
597\end{array}$ & $\begin{array}{l}3.9 \\
6.1 \\
9.4 \\
21.9 \\
7.4\end{array}$ & $\begin{array}{l}440 \\
565 \\
391 \\
90 \\
1486\end{array}$ & $\begin{array}{l}5.5 \\
5.8 \\
11.8 \\
24.4 \\
8.4\end{array}$ \\
\hline Soft, intermediate & $\begin{array}{l}50-59 \\
60-69 \\
70-79 \\
80+ \\
\text { Total }\end{array}$ & $\begin{array}{l}285 \\
334 \\
211 \\
58 \\
889\end{array}$ & $\begin{array}{l}0 \\
0.90 \\
0.47 \\
0 \\
0.45\end{array}$ & $\begin{array}{l}155 \\
231 \\
180 \\
32 \\
597\end{array}$ & $\begin{array}{l}0 \\
0.43 \\
1.1 \\
0 \\
0.50\end{array}$ & $\begin{array}{l}440 \\
565 \\
391 \\
90 \\
1486\end{array}$ & $\begin{array}{l}0 \\
0.71 \\
0.77 \\
0 \\
0.47\end{array}$ \\
\hline Cannot grade & $\begin{array}{l}50-59 \\
60-69 \\
70-79 \\
80+ \\
\text { Total }\end{array}$ & $\begin{array}{l}285 \\
334 \\
211 \\
58 \\
889\end{array}$ & $\begin{array}{l}0 \\
0 \\
0 \\
0 \\
0\end{array}$ & $\begin{array}{l}155 \\
231 \\
180 \\
32 \\
597\end{array}$ & $\begin{array}{l}1.3 \\
0 \\
0.56 \\
0 \\
0.50\end{array}$ & $\begin{array}{l}440 \\
565 \\
391 \\
90 \\
1486\end{array}$ & $\begin{array}{l}0.45 \\
0 \\
0.26 \\
0 \\
0.20\end{array}$ \\
\hline
\end{tabular}

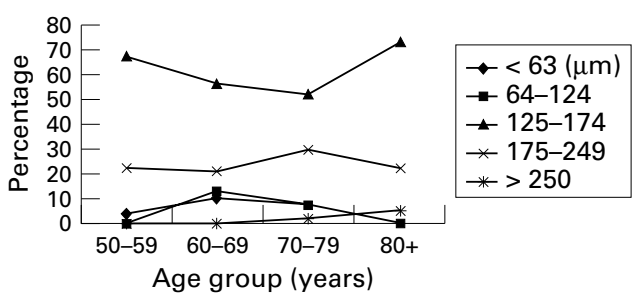

Figure 1 The frequency of the drusen size by age in the Hisayama study. There were no significant differences in the frequency of these larger drusen in terms of sex or age.

DEFINITION OF AGE RELATED MACULOPATHY

The graders, masked to subject information, used the International ARM Epidemiological Study Group grading protocol and the grids of the Wisconsin Age Related Maculopathy Grading system, described previously. ${ }^{16}{ }^{17}$ The Wisconsin Age Related Maculopathy Grading System grid was adapted to the magnification of the camera. This protocol divides ARM into early and late stages. Early ARM is defined as the presence of drusen or retinal pigment epithelium (RPE) pigmentary abnormalities within the grid in the absence of late ARM in either eye. (This definition of early ARM was used in both the studies in Beaver Dam, in Wisconsin, and in Blue Mountains eye studies.) The protocol distinguishes two types of late ARM-neovascular AMD, also termed "wet AMD," and geographic atrophy of the $\mathrm{RPE}$ in the absence of neovascular AMD, also termed "dry AMD." Neovascular AMD includes serous or haemorrhagic detachment of the retinal pigment epithelium or sensory retina, and the presence of subretinal or subretinal pigment epithelium haemorrhages or subretinal fibrous scar tissue. Dry AMD is characterised by sharply edged, roughly round or oval areas of RPE hypopigmentation, with clearly visible choroidal vessels. The minimum area of geographic atrophy is a circle $175 \mu \mathrm{m}$ diameter or larger.

The age and sex specific frequencies of each ARM grade, as well as of early and late ARM, were determined. The analysis is based on people with gradable photographs in either eye providing the clearest macular characteristics detected by stereoscopic eye examination. Interobserver and intraobserver variability were analysed by kappa statistics. Agreement levels were moderate to substantial for most features.

\section{DATA COLLECTION}

Blood pressures were measured three times at each examination, and the average values were used for the analysis. Diabetes was revealed by medical history, glucose levels, or a $75 \mathrm{~g}$ oral glucose tolerance test given to most of the subjects. Body weight and height were measured in light clothing without shoes, and body mass index (BMI) was calculated. Percentage body fat was measured and calculated using a body fat analyser (TBF-202, Tanita, Tokyo, Japan). Information on smoking habits was obtained with the use of a standard questionnaire. 
Table 4 The prevalence of pigmentary abnormalities by age and sex in the Hisayama Study, after excluding patients with late age related maculopathy in either eye

\begin{tabular}{|c|c|c|c|c|c|c|c|}
\hline & \multirow[b]{2}{*}{ Age } & \multicolumn{2}{|c|}{ Female } & \multicolumn{2}{|c|}{ Male } & \multicolumn{2}{|l|}{ Total } \\
\hline & & No & $\%$ Freq & No & $\%$ Freq & No & $\%$ Freq \\
\hline Hyperpigmentation & $\begin{array}{l}50-59 \\
60-69 \\
70-79 \\
80+ \\
\text { Total }\end{array}$ & $\begin{array}{l}285 \\
334 \\
211 \\
58 \\
889\end{array}$ & $\begin{array}{l}0 \\
0.30 \\
0.95 \\
1.7 \\
0.45\end{array}$ & $\begin{array}{l}155 \\
231 \\
180 \\
32 \\
597\end{array}$ & $\begin{array}{l}0 \\
2.6 \\
2.8 \\
0 \\
1.8\end{array}$ & $\begin{array}{l}440 \\
565 \\
391 \\
90 \\
1486\end{array}$ & $\begin{array}{l}0 \\
1.2 \\
1.8 \\
1.1 \\
1.0\end{array}$ \\
\hline Hypopigmentation & $\begin{array}{l}50-59 \\
60-69 \\
70-79 \\
80+ \\
\text { Total }\end{array}$ & $\begin{array}{l}285 \\
334 \\
211 \\
58 \\
889\end{array}$ & $\begin{array}{l}2.8 \\
0.90 \\
1.4 \\
0 \\
1.6\end{array}$ & $\begin{array}{l}155 \\
231 \\
180 \\
32 \\
597\end{array}$ & $\begin{array}{l}3.2 \\
3.9 \\
1.1 \\
6.3 \\
3.0\end{array}$ & $\begin{array}{l}440 \\
565 \\
391 \\
90 \\
1486\end{array}$ & $\begin{array}{l}3.0 \\
2.1 \\
1.3 \\
2.2 \\
2.2\end{array}$ \\
\hline Either type & $\begin{array}{l}50-59 \\
60-69 \\
70-79 \\
80+ \\
\text { Total }\end{array}$ & $\begin{array}{l}285 \\
334 \\
211 \\
58 \\
889\end{array}$ & $\begin{array}{l}2.8 \\
1.2 \\
2.4 \\
1.7 \\
2.0\end{array}$ & $\begin{array}{l}155 \\
231 \\
180 \\
32 \\
597\end{array}$ & $\begin{array}{l}3.2 \\
6.5 \\
3.9 \\
6.3 \\
4.9\end{array}$ & $\begin{array}{l}440 \\
565 \\
391 \\
90 \\
1486\end{array}$ & $\begin{array}{l}3.0 \\
3.4 \\
3.1 \\
3.3 \\
3.2\end{array}$ \\
\hline
\end{tabular}

Table 5 The prevalence of geographic atrophy or neovascular AMD in the Hisayama study

\begin{tabular}{|c|c|c|c|c|c|c|c|}
\hline & \multirow{2}{*}{$\begin{array}{l}\text { Age } \\
\text { (years) }\end{array}$} & \multicolumn{2}{|c|}{ Female } & \multicolumn{2}{|l|}{ Male } & \multicolumn{2}{|l|}{ Total } \\
\hline & & No & $\%$ Freq & No & $\%$ Freq & No & $\%$ Freq \\
\hline \multirow[t]{5}{*}{ Geographic atrophy } & $50-59$ & 285 & 0 & 155 & 0 & 440 & 0 \\
\hline & $60-69$ & 334 & 0 & 231 & 0.87 & 565 & 0.35 \\
\hline & $70-79$ & 211 & 0 & 180 & 0 & 391 & 0 \\
\hline & $80+$ & 58 & 0 & 32 & 3.1 & 90 & 1.1 \\
\hline & Total & 889 & 0 & 597 & 0.50 & 1486 & 0.20 \\
\hline \multirow{5}{*}{ Neovascular AMD } & $50-59$ & 285 & 0.70 & 155 & 0 & 440 & 0.45 \\
\hline & $60-69$ & 334 & 0.30 & 231 & 1.7 & 565 & 0.88 \\
\hline & $70-79$ & 211 & 0 & 180 & 1.1 & 391 & 0.51 \\
\hline & $80+$ & 58 & 0 & 32 & 3.1 & 90 & 1.1 \\
\hline & Total & 889 & 0.34 & 597 & 1.2 & 1486 & 0.67 \\
\hline
\end{tabular}

Table 6 Age related maculopathy by sex in the Hisayama study

\begin{tabular}{|c|c|c|c|c|c|c|c|c|c|}
\hline \multirow[b]{2}{*}{ Age } & \multicolumn{3}{|c|}{ Female } & \multicolumn{3}{|c|}{ Male } & \multicolumn{3}{|l|}{ Total } \\
\hline & No & $\begin{array}{l}\text { Early } \\
(\%)\end{array}$ & $\begin{array}{l}\text { Late } \\
(\%)\end{array}$ & No & $\begin{array}{l}\text { Early } \\
(\%)\end{array}$ & $\begin{array}{l}\text { Late } \\
(\%)\end{array}$ & No & $\begin{array}{l}\text { Early } \\
(\%)\end{array}$ & $\begin{array}{l}\text { Late } \\
(\%)\end{array}$ \\
\hline $50-59$ & 285 & 9.1 & 0.70 & 155 & 9.0 & 0 & 440 & 9.1 & 0.45 \\
\hline $60-69$ & 334 & 8.1 & 0.30 & 231 & 13.4 & 2.6 & 565 & 10.3 & 1.2 \\
\hline $70-79$ & 211 & 17.5 & 0 & 180 & 16.1 & 1.1 & 391 & 16.9 & 0.51 \\
\hline $80+$ & 58 & 27.6 & 0 & 32 & 28.1 & 6.3 & 90 & 27.8 & 2.2 \\
\hline Total & 889 & 11.9 & 0.33 & 597 & 13.9 & 1.7 & 1486 & 12.7 & 0.87 \\
\hline
\end{tabular}

STATISTICAL METHODS

The Mann-Whitney $U$ test or the $\chi^{2}$ test with Yates's correction was used to detect differences in proportions. BMDP statistical software (BMDP Statistical Software, University of California Press, Los Angeles, CA, USA) was used for the logistic regression analysis on a SPARK station 20; age was entered as a categorical variable. All confidence intervals (CI) presented are $95 \%$.

\section{Results}

The mean age of the participants was 65.2 years. Men comprised $40.1 \%$ of the group, women $59.9 \%$. This sex ratio was the same as that for all Hisayama inhabitants aged 50 years or older. The mean systolic blood pressure was $137.2 \mathrm{~mm} \mathrm{Hg}$, the mean diastolic pressure $78.1 \mathrm{~mm} \mathrm{Hg}$. The mean percentage body fat was $24.8 \%$, and the mean body mass index (BMI) was $23.2 \mathrm{~kg} / \mathrm{m}^{2}$. Smokers comprised $18.1 \%$ of the participants. The prevalence of diabetes was $18.2 \%$ (Table 1). From 1486 participants, five fundus photographs could not be obtained owing to technical failure (two males and three females, mean age; 62.3). All of them could be graded by direct and indirect ophthalmic examinations.

Drusen were present in the macular area in $9.6 \%$ of the total number of participants. The frequency of any drusen was the same for the men and women. Age was strongly associated with an increasing prevalence of drusen in both the men and women $(\mathrm{p}<0.001)$ (Table 2$)$. The relative odds ratio of having drusen was 6.6 ( $95 \%$ CI, 3.84 to 11.5 ) in people 80 years of age or older compared with those 50-59 years of age. Distinct soft drusen were the most frequent largest drusen present within the macular area at all ages (Table 3). The larger drusen sizes $(125-174 \mu \mathrm{m}$ in diameter) were the most frequent type among all age groups. There were no significant differences in drusen size for any age or either sex (Fig 1).

Hyperpigmentation and/or hypopigmentation of RPE was present in $3.2 \%$. Hyperpigmentation was identified in $1.0 \%$, hypopigmentation in $2.1 \%$. The prevalence of hyperpigmentation among the men was significantly higher than it was among the women ( $p$ $<0.05$; odds ratio 4.15; CI, 1.28 to 13.5 ). Hypopigmentation tended to be seen more frequently in the men than in the women, but the difference was not significant. Overall, retinal pigmentary abnormalities were found more commonly in the men than in the women ( $\mathrm{p}$ $<0.01$, odds ratio 2.47 ; CI, 1.34 to 4.55 ). There were no significant differences in frequency of hyperpigmentation and/or hypopigmentation with respect to age (Table 4).

Geographic atrophy was present in the macular area in three men $(0.2 \%)$ (Table 5$)$. Neovascular AMD was found in $10(0.67 \%)$. Of these, only one $(0.07 \%)$ had bilateral neovascular AMD. There were no significant differences between the prevalence of neovascular AMD for any ages. The men had a significantly higher frequency of neovascular AMD than the women $(1.2 \%$ v $0.34 \%$, p $<0.01$, odds ratio 5.16; CI, 1.41 to 18.9 ).

The frequencies of the different stages of ARM are shown in Table 6 . Overall, $12.7 \%$ of the population had some signs of early ARM in the macular area. The frequency of early ARM increased significantly with age $(p<0.01)$. The relative odds ratio of having early ARM was 3.02 ( $95 \%$ CI, 1.5 to 6.1 ) in those 80 years of age or older compared with those 50-59 years old. There were no differences between the prevalence of early ARM by sex. The percentage frequency of late ARM was $0.87 \%$ overall. The percentage frequency of late ARM among the men was significantly higher than it was among the women $(0.33 \% v 1.7 \%, \mathrm{p}<0.01$, odds ratio 5.16 , CI 1.41 to 18.9 ). Although the frequency of late ARM tended to increase with age among the men $(\mathrm{p}=0.08)$, the numbers were too small to show a definite trend. There were no differences between the frequency of late ARM for any ages, for the women, and overall.

Among the participants with unilateral late ARM, the uninvolved eyes were more likely to have advanced ARM characteristics than the uninvolved eyes of the participants with no characteristics of late stage ARM (Table 7). 
Table 7 Relation of early age related maculopathy characteristics to late age related maculopathy in the Hisayama study

\begin{tabular}{|c|c|c|c|c|c|}
\hline \multirow[b]{2}{*}{ Characteristic } & \multicolumn{2}{|c|}{$\begin{array}{l}\text { Non-involved eye of } \\
\text { people with unilateral } \\
\text { late ARM }\end{array}$} & \multicolumn{2}{|c|}{$\begin{array}{l}\text { Right eye of people with } \\
\text { neither eye involved } \\
\text { with late ARM }\end{array}$} & \multirow[b]{2}{*}{$p$ Value } \\
\hline & Case & $\%$ & Case & $\%$ & \\
\hline Soft distinct drusen present & 1 & 9.1 & 91 & 6.1 & 0.7 \\
\hline Hyper/hypopigmentation present & 4 & 36.3 & 25 & 1.7 & $<0.001$ \\
\hline Early ARM present & 5 & 45.5 & 148 & 10.1 & $<0.001$ \\
\hline
\end{tabular}

Table 8 The relation of blood pressure, body mass index and percentage body fat, and age related maculopathy in the Hisayama study

\begin{tabular}{llllllll}
\hline & \multicolumn{2}{l}{ Case } & & & \multicolumn{2}{l}{ Control } & \\
\cline { 2 - 3 } & No & Mean $(S D)$ & & No & Mean (SD) & p Value \\
\hline Systolic blood pressure (mm Hg) & 202 & $139.5(22.9)$ & & 1284 & $136.8(21.6)$ & 0.19 \\
Diastolic blood pressure (mm Hg) & 202 & $77.4(10.4)$ & & 1284 & $78.2(10.8)$ & 0.54 \\
Body mass index $\left(/ \mathrm{kg} / \mathrm{m}^{2}\right)$ & 202 & $23.0(3.3)$ & & 1284 & $23.2(3.2)$ & 0.66 \\
Percentage body fat & 202 & $23.5(7.9)$ & & 1284 & $25.0(7.0)$ & 0.002
\end{tabular}

Table 9 The relation of diabetes status, smoking status, and age related maculopathy in the Hisayama study

\begin{tabular}{|c|c|c|c|c|c|c|c|}
\hline & & \multicolumn{2}{|c|}{ Case } & \multicolumn{2}{|c|}{ Control } & \multirow[b]{2}{*}{ OR } & \multirow[b]{2}{*}{$95 \% C I$} \\
\hline & & $\mathrm{No}$ & $\%$ & No & $\%$ & & \\
\hline \multirow{2}{*}{ Diabetes status } & Absent & 163 & 11.1 & 1031 & 70.4 & & \\
\hline & Present & 39 & 2.7 & 232 & 15.8 & 1.06 & $(0.61-1.83)$ \\
\hline \multirow[t]{2}{*}{ Current smoking } & Absent & 164 & 11.2 & 1032 & 70.4 & & \\
\hline & Present & 38 & 2.6 & 231 & 15.8 & 1.04 & $(0.44-2.41)$ \\
\hline
\end{tabular}

Systolic blood pressure, diastolic blood pressure, and body mass index were not related to the presence of ARM. The percentage body fat was significantly lower among those with ARM ( $\mathrm{p}<0.01$ ), but there were no differences between the presence of ARM and percentage body fat after controlling for age (Table 8). There were no differences between the presence of diabetes mellitus and ARM in general (odds ratio 1.06 , CI 0.61 to 1.83 ), early ARM (odds ratio 1.05 , CI 0.56 to 1.99 ), and late ARM (odds ratio 1.32 , CI 0.36 to 4.83 ). Current tobacco smoking was not associated with ARM (odds ratio 1.04 , CI 0.44 to 2.41 ), early ARM (odds ratio 1.10 , CI 0.67 to 1.8 ), or late ARM (odds ratio 0.37, CI 0.02 to 8.46) (Table 9).

\section{Discussion}

The current study was performed as part of a prospective cohort study in a representative Japanese population, the Hisayama study, which has been carried out since $1961 .^{13}$

The results of this study generally can be compared with those found in other population based studies, but some methodological issues should be mentioned. The procedure of taking and evaluating fundus photographs was standardised. The photographs were examined by experienced graders in a masked fashion, and the reproducibility of the clinical and photographic protocols was evaluated throughout the study. We used monoscopic fundus photographs produced according to previously described methods with minor modifications. ${ }^{4} 915$ Although reliance on monoscopic photographs theoretically could result in underestimation of the prevalence of ARM by missing subtle early macular changes, especially retinal pigment epithelium detachment, we sought to minimise this potential shortcoming by having well trained ophthalmologists examine the participants' eyes by stereoscopic indirect ophthalmoscopy. Almost all the fundus photographs were of acceptable quality, although the ones taken of older eyes tended to be worse because of media opacities. Again, however, the inclusion of stereoscopic indirect ophthalmoscopy in the examination tended to compensate for this potential deficiency.

The prevalence of any form of late ARM was $0.87 \%$, that of neovascular AMD $0.67 \%$, and that of geographic atrophy $0.20 \%$. Since we graded fundus characteristics according to the International ARM Epidemiological Study Group grading protocol, ${ }^{16}$ a modification of the Wisconsin age related maculopathy grading system (WARMGS), ${ }^{17}$ the Hisayama findings can be usefully compared with other population studies using this grading system.

The prevalence rate of total late ARM we found was lower than that reported in studies performed in white populations (Beaver Dam Eye Study: $1.6 \%$, Rotterdam Study: $1.7 \%$, Blue Mountain Eye Study: 1.9\%), but was slightly higher than that found in the Barbados Eye Study $(0.6 \%)$ and the Baltimore Eye Survey $(0.22 \%)$, both of which focused on black populations. $^{78}$ The prevalence of geographic atrophy was lower than that of neovascular AMD in our study, as was the case in studies performed in white populations. ${ }^{34}$

Although in these other studies the prevalence of late ARM significantly increased with age, we found no such correlation. This difference may have resulted from the relative low number of people with late ARM changes in our study.

Although we found a significantly higher prevalence of late ARM among men than among women, there was no such differences reported in the Rotterdam Study and Blue Mountain Eye Study. In the Beaver Dam Eye Study, women 75 years of age or older had a significantly higher frequency of exudative macular degeneration than similarly aged men. The reason is not clear. Yuzawa et $a l,{ }^{12}$ who estimated the prevalence of exudative AMD based on the number of patients undergoing medical care in Japan, found that exudative AMD was significantly more prevalent among men than among women. ${ }^{12}{ }^{18}$ This finding is consistent with ours.

Our study, as well as the Barbados Eye Study $^{7}$ and the Blue Mountain Eye Study, found that the prevalence of early ARM significantly increased with age. However, we found no differences between the prevalence of ARM among the men and the women. In our study $12.7 \%$ had early ARM changes, a lower figure than that reported in the Barbados Eye Study $(23.5 \%)$ and in the Blue Mountain Eye Study (28.0\%).

Drusen have been more commonly observed in white people (Beaver Dam Eye Study, 86\%) than in black people (Barbados Eye Study, $66.2 \%$ ). This difference was also reported in a racial differences study in the Third National Health and Nutrition Examination Survey 
(The NHANES III) ${ }^{9}$ and in the Baltimore Eye Survey (whites: $21.7 \%$, blacks: $18.8 \%){ }^{8}$ In our study, the prevalence of any type of drusen was $9.6 \%$. Although this prevalence was lower than that reported in other studies, in our study, as well as in these others, the prevalence of drusen markedly increased with age.

Hyperpigmentation and/or hypopigmentation were seen in $3.2 \%$ of the Hisayama population. This prevalence is lower than that found in white people (Beaver Dam Eye Study: $13.1 \%$, Blue Mountain Eye Study: $12.6 \%$, Rotterdam Study: $7.2 \%$ ), but higher than that found among black people (Baltimore Eye Survey; $1.84 \%$ ). It was seen more frequently in men than in women in our study, in the Rotterdam Study (odds ratio: 1.6 for hypopigmentation, 1.5 for hyperpigmentation), and in the Beaver Dam Eye Study among those 65 years old or older.

Some studies have reported racial differences in the prevalence of ARM..$^{7-10}$ These reports have suggested that neovascular AMD is more prevalent in white people than in black people, especially among older individuals. The NHANES III found a higher prevalence of ARM in non-Hispanic whites $(9.3 \%)$ than in non-Hispanic black people $(7.4 \%)$ and Mexican-Americans (7.1\%). ${ }^{9}$ The Baltimore Eye Survey reported no differences in the prevalence of early ARM in white and black people but found that late ARM is more prevalent in older white people $(1.91 \%)$ than in older black people $(0.19 \%){ }^{8}$

To our knowledge, there have been no studies of the prevalence of ARM among any Asian population except for Wu's investigation in China, ${ }^{11}$ which found a prevalence of $7.67 \%$, with no significant differences among three ethnic groups (Han, Uighur, and Tibetan). This prevalence is about 10 times higher than what we found among our representative Japanese population. This was probably because his study graded fundus characteristics based on the Framingham Eye Study, ${ }^{1}$ a different method from the one we used. The prevalence of early and late ARM in our study is much lower than that found in studies of white populations, while the late ARM changes we found were slightly more frequent than those reported in some studies of black populations. The reason for different prevalences among different races is not clear. However, Friedman et al in the Baltimore Eye Survey speculated that white people are genetically predisposed to have more severe maculopathy than black people. ${ }^{8}$

Hisayama study used non-stereoscopic $45^{\circ}$ fundus photographs to grade of ARM. It is known that age related maculopathy, especially early ARM, is less likely to be detected by grading of fundus photographs than grading of $30^{\circ}$ stereoscopic fundus photographs. It is supposed that grading of stereoscopic colour fundus photographs probably explain, in part, the lower frequencies of early and late ARM frequencies in Hisayama study compared with the frequencies among white people in Western countries.

It is known that smoking (ever/never smoked) is a risk factor for late ARM. ${ }^{18-20}$ However, we found no association between current smoking and ARM, probably because we found few people with late ARM changes, and we did not study former smokers or level of smoking. Further investigation is needed to clarify the relation between smoking and ARM in Japan.

In conclusion, our study suggested that early and late stage ARM is less common among the Japanese than among white people in Western countries, while late stage ARM is more common among the Japanese than among black people.

This work was supported in part by grants in aid No 11002994 from the Scientific Research from Ministry of Education, Science, Culture and Sports of the Japanese Government, the Japan Society for the Promotion of Science (Tokyo), the Japan
Eye Bank Association (Tokyo). The authors thank Yuko Eye Bank Association (Tokyo). The authors thank Yuko
Nishioka, MD, and Naoko Kinukawa, PhD, for their assistance in analyses of the data and Dr Kenneth W Parker for editorial in analyses

1 Leibowitz HM, Krueger DE, Maunder LR, et al. The Framingham Eye Study monograph: an ophthalmological and epidemiological study of cataract, glaucona, diabetic retinpathy, macular degeneration, and visual acuity in a general 1980;24:335-610.

2 Maguire MG. Natural history. In: Berger JW, Fine SL, Maguire MG, eds. Age-related macular degeneration. St Louis: Geff Greenwood, 1999:17-30

3 Klein R, Klein BE, Linton KL. Prevalence of age-related maculopathy. The Beaver Dam Eye Study. Ophthalmology 1992;99:933-43.

4 Vingerling JR, Dielemans I, Hofman A, et al. The prevalence of age-related maculopathy in the Rotterdam Study. Ophthalmology 1995;102:205-10

5 Mitchell P, Smith W, Attebo K, et al. Prevalence of age-related maculopathy in Australia. The Blue Mountains Eye Study. Ophthalmology 1995;102:1450-60.

6 Bressler NM, Bressler SB, West SK, et al. The grading and prevalence of macular degeneration in Chesapeake Bay watermen. Arch Ophthalmol 1989;107:847-52.

7 Schachat AP, Hyman L, Leske MC, et al. Features of age-related macular degeneration in a black population. The Barbados Eye Study Group. Arch Ophthalmol 1995; 113: $728-35$.

113:728-35.
8 Friedman DS, Katz J, Bressler NM, et al. Racial differences in the prevalence of age-related macular degeneration: the Baltimore Eye Survey. Ophthalmology 1999;106:1049-55.

9 Klein R, Rowland ML, Harris MI. Racial/ethnic differences in age-related maculopathy. Third National Health and Nutrition Examination Survey. Ophthalmology 1995;102: 371-81.

10 Klein R, Klein BE, Jensen SC, et al. Age-related maculopathy in a multiracial United States population: the National Health and Nutrition Examination Survey III. Ophthalmology 1999;106:1056-65.

11 Wu LH. Study of aging macular degeneration in China. $f p n$ f Ophthalmol 1987;31:349-67.

12 Yuzawa M, Tamakoshi A, Kawamura T, et al. Report on the nationwide epidemiological survey of exudative age-related macular degeneration in Japan. Int Ophthalmol 1997;21:1-3.

13 Ohmura T, Ueda K, Kiyohara Y, et al. Prevalence of type 2 (non-insulin-dependent) diabetes mellitus and impaired glucose tolerance in the Japanese general population: the Hisayama Study. Diabetologia 1993;36:1198-203.

14 Iwamoto H, Kiyohara Y, Fujishima M, et al. Prevalence of intracranial saccular aneurysms in a Japanese community based on a consecutive autopsy series during a 30-year observation period. The Hisayama study. Stroke 1999;30: 1390-5.

15 Klein R, Meuer SM, Moss SE, et al. Detection of drusen and early signs of age-related maculopathy using a nonmydriatic camera and a standard fundus camera. Ophthalmoldriatic camera and a stan

16 Bird AC, Bressler NM, Bressler SB, et al. An international classification and grading system for age-related maculopathy and age-related macular degeneration. The International ARM Epidemiological Study Group. Surv Ophthalmol 1995;39:367-74

17 Klein R, Davis MD, Magli YL, et al. The Wisconsin age-related maculopathy grading system. Ophthalmology 1991;98:1128-34.

18 Tamakoshi A, Yuzawa M, Matsui M, et al. Smoking and neovascular form of age related macular degeneration in late middle aged males: findings from a case-control study in Japan. Research Committee on Chorioretinal Degenerations. Br f Ophthalmol 1997;81:901-4.

19 Smith W, Mitchell P, Leeder SR. Smoking and age-related maculopathy. The Blue Mountains Eye Study. Arch Ophthalmol 1996;114:1518-23.

20 Vingerling JR, Hofman A, Grobbee DE, et al. Age-related macular degeneration and smoking. The Rotterdam Study. Arch Ophthalmol 1996;114:1193-6. 